proportion of the alkaloid is converted by the liver of the host into an inactive metabolite. The amount of alkaloid converted in vivo is probably much greater than is converted in liver suspensions.

This metabolizing of quinine, with consequent loss of antimalarial activity, might be borne in mind in designing new derivatives or synthetic products of the cinchona type. We have indications, for example, that, while quinidine is metabolized similarly to quinine, einchonine and cinchonidine are not metabolized by liver suspensions (chick liver). Mepacrine and plasmoquin also appear to be unchanged by rabbit liver suspensions.

Wellcome Laboratories of Tropical Medicine,

P. B. MarshatI.

183 Euston Road, London, N.W.1. July 23. ${ }^{2}$ Kelsey. F. E., Geiling, E. M. K., Oldham, F. K., and Dearborn,

2 Mead, J., and Koepfli, J. B., J. Biol. Chem., 154, 507 (1944).

\section{A Convenient 'Bridge' for Two-Solution Cells}

IN the setting up of two-solution cells for the measurement of electromotive force, it is not easy to establish a satisfactory junction between the two liquids, so tedious and cumbersome devices, such as the agar jelly bridge, are commonly in use. The difficulty may be overcome, and stable and correct junction potentials obtained within a fow seconds, by the simple device of using for the bridge a strip of filter paper, the ends of which dip into the two solutions, which may conveniently be contained in two small beakers resting on a block of paraffin wax.

If the filter paper be used dry, a liquid-liquid junction is immediately set up by capillary rise.

If it is desired to use a third liquid for the bridge, a strip of paper may be soaked in this, and the excess removed before use by pressing between dry filter papers.

If a galvanometer of high sensitivity is in use, a single strand of bleached cotton yarn will form a satisfactory bridge, and the device may be further improved so as to give readings which remain stable indefinitely, by drawing the yarn through a glass U-tube, so as to shield it from evaporation.

College of Technology,

Manchester, 1. July 6.

\section{Chromium Oxide as a Promoter in Catalysts for the Fischer-Tropsch Synthesis}

THE economics of synthesis of hydrocarbons by the Fischer-Tropsch process may be improved by using (1) cheaper catalytic material, (2) water gas as such in place of enriched synthesis gas $\left(\mathrm{CO}: \mathrm{H}_{2}\right.$ as $1: 2$ ) and (3) medium pressures instead of atmospheric pressures. A cheap iron-copper catalyst prepared by Ghosh and Sen ${ }^{1}$ gave $86 \mathrm{gm}$. of hydrocarbons per cubic metre of gas and has since been developed by Japanese investigators ${ }^{2}$. The fact that chromium oxide possesses very high adsorption for hydrogen at $200^{\circ}$ has been taken advantage of in preparing a catalyst $(\mathrm{CO}=34$ per cent; $\mathrm{Cu}=4$ per cent; $\mathrm{ThO}_{2}=2 \cdot 3$ per cent; $\mathrm{Ce}_{2} \mathrm{O}_{3}=0.24$ per cent; $\mathrm{Cr}_{2} \mathrm{O}_{3}=4 \cdot 6$ per cent; rest kieselguhr) which has been found to be romarkably steady, and yields with water-gas at a pressure of 5 atmospheres and $205^{\circ} \mathrm{C}$., $160 \mathrm{gm}$. of liquid hydrocarbons per cubic metre with a space velocity of 600 c.c. per hour per c.c. of catalyst.

\section{Indian Institute of Science, Bangalore, India.}

J. C. Ghosh.

S. L. SASTRY. Feb. 28, 1944.

[Publication delayed for security reasons. EDITons.]

${ }^{1} J$. Indian Chem. Soc., 12, 53 (1935).

${ }^{2}$ T'suneoka, et al., Sci. Papers Inst. Phys. Chem. Res., Tokyo, 35, 330 $(1938-39) ; 38,118(1940) ; 38,184(1941)$.

\section{Darkened Violet in Colour Vision}

EDRIDGE-GREeN ${ }^{1}$ has directed attention to the shortening of the violet end of the spectrum which he found in some persons. A test of sensitivity to red, orange, yellow, yellow-green, green, blue-green, blue, violet and purple was carried out on $103 \mathrm{men}$ and 104 women, excluding all red-green blind and red or green anomalous subjects. The experiment was performed with a form of colorimeter using the Ilford Monochromatic filters (601-8) and purple (501). Relative brightness was measured as well as sensitivity to hue.

Three women and three men had the violet darkened by more than three times the standard deviation of brightness levels of violet for the remaining subjects, which shows that the darkening was not due to normal variation (or random error). A number had slight darkening of violet and blue, but while there was a correlation of +0.337 between blue weakness and darkening of blue, the correlation between violet weakness and darkening of violet was not significant. This means that on the whole there was a tendency for blue to be darkened when it was weakened, while violet was as often brighter as darker when there was loss of sensitivity to its hue.

In this test there were about 10 per cent who had marked yellow-blue weaknesses and were not redgreen defectives (while the red-green blind are rarely weak in yellow or blue). This confirmed the result of previous tests. The yellow-blue weak subjects were not sufficiently defective to be called blueyellow blind and they were not consistently detected by tests in general use; but they had sometimes suspected a weakness of colour-sensitivity in themselves. Six of the yellow-blue weak subjects were those with darkened violet. Five of them were weak in yellow as well as in blue, and one in blue rather than in yellow. All were weaker in yellow and/or blue than in violet, but not weaker than the yellowblue weak in whom violet was normally bright.

Equality of proportion for both sexes suggests that blue-yellow weakness and darkened violet are not sex-linked characters, a conclusion supported by genealogical data. Darkening of violet appears to be about as common in each sex as the familiar darkening of red (as in protanopes) among men. Just as, in my experience with detailed tests on about seventy red-green defectives, the protanopes are not 'red-blind' but are red-green defectives with darkened red as an additional character, so those with darkened violet are not 'blue-blind' but are yellow-blue defectives with violet and blue of diminished brightness.

Thus, although there are, as Walls ${ }^{2}$ has predicted, two classes of blue-defectives, namely, those with 